\title{
Ovarian, Fallopian Tube, and Primary Peritoneal Carcinoma pT1a TNM Finding v8
}

National Cancer Institute

\section{Source}

National Cancer Institute. Ovarian, Fallopian Tube, and Primary Peritoneal Carcinoma

pT1a TNM Finding v8. NCI Thesaurus. Code C139943.

Ovarian, fallopian tube, or primary peritoneal carcinoma with tumor limited to one ovary (capsule intact) or fallopian tube surface; no malignant cells in ascites or peritoneal washings. (from AJCC 8th Ed.) 